\title{
Pacientes con traumas faciales ocurridos en accidentes con motocicletas en Brasil
}

Patients with facial trauma arising from motorcycle accidents in Brazil

Pacientes com traumas faciais decorrentes de acidentes motociclísticos no Brasil

\author{
Ana Ruth Lima CORDEIRO \\ Gardênia Moura de SOUSA ${ }^{1}$ \\ Ana Larisse Carneiro PEREIRA ${ }^{2}$ \\ Jefferson David Melo de MATOS $^{3}$ \\ André Guimarães RODRIGUES ${ }^{4}$ \\ Valdir Cabral ANDRADE ${ }^{5}$ \\ Francisco Jadson LIMA ${ }^{6}$
}

\author{
Cirurgiã-Dentista, Departamento de Odontología, Centro Universitario Leão Sampaio UNILEÃO, 63022-145 Juazeiro do Norte - CE, Brasil \\ ${ }^{2}$ Mestranda, Programa de Pós-Graduação em Ciências Odontológicas, Área de concentração em Prótese Dentária, \\ Universidade Federal do Rio Grande do Norte UFRN 59078-970 Natal-RN, Brasil \\ ${ }^{3}$ Mestrando, Programa de Pós-Graduação em Odontologia Restauradora, Departamento de Materiais Odontológicos e Prótese, Universidade Estadual Paulista \\ (Unesp), Instituto de Ciência e Tecnologia, São José dos Campos, 12245-000 São José dos Campos-SP, Brasil \\ ${ }^{4}$ Cirurgião-Dentista, Universidade de Vila Velha (UVVES), 29102-920 Vila Velha-ES Brasil \\ ${ }_{5}^{5}$ Professor Adjunto, Departamento de Odontología, Universidade Federal de Juiz de Fora UFJF - Campus Governador Valadares, \\ 35010-180 Governador Valadares - MG, Brasil \\ ${ }^{6}$ Professor, Departamento de Odontología, Centro Universitário Leão Sampaio UNILEÃO, 63022-145 Juazeiro do Norte - CE, Brasil
}

\section{Resumen}

Objetivo: El objetivo del presente estudio fue describir el perfil de los pacientes con traumas de cara ocurridos en accidentes con motocicletas en Brasil. Métodos: El estudio consistió en una revisión integrativa basada en la literatura. Las bases de datos consultadas fueron el Scientific Electronic Library on-line (SciELO) y la National Library of Medicine (PubMed), utilizando los siguientes descriptores: Accidentes de Tránsito, Epidemiología y Traumatismos Faciales, de acuerdo con las terminologías consultadas en los Descriptores en Ciencias de la Salud (DECS-BIREME). La búsqueda consideró las publicaciones publicados entre los años 1995 a 2015. Resultados: En cuanto al sexo masculino, los individuos jóvenes prevalecieron en el 100\% de los estudios. Sólo el 23,5\% de los estudios presentaron el nivel de escolaridad de los pacientes, la condición de salud de las víctimas en el 64,7\% de los casos se presentó con fracturas y traumas. La región más traumatizada fue la cara en el $41,1 \%$ de los casos, en relación al tipo de lesión en el $17 \%$ de los casos se dio por trauma y el $6,4 \%$ se dio por contusiones. En el 24,5\% de los estudios las víctimas hicieron uso de bebidas alcohólicas y el 29,4\% no utilizaba el casco en el momento del accidente. Conclusión: Ante ello es necesario que estudios que identifiquen o tracen el perfil de esos individuos, determinando tipo de accidentes y compromisos sean realizados de forma más detallada, para producir subsidios necesarios para la planificación de políticas públicas en salud y promoción de medidas preventivas.

Descriptores: Accidentes de Tránsito; Epidemiología; Traumatismos Faciales.

\section{Abstract}

Purpose: The aim of the present study was to describe the profile of patients with facial trauma due to motorcycle accidents in Brazil. Methods: The study consisted of an integrative review based on the literature. The databases consulted were the Scientific Electronic Library Online (SciELO) and National Library of Medicine (PubMed), using the following descriptors: Accidents, Traffic AND Epidemiology AND Facial Injuries, according to the terminologies consulted in the Descriptors in Sciences of Health (DECS-BIREME). The search considered the publications from the year 1995 to 2015 . Results: As for males, young individuals prevailed in $100 \%$ of the studies. Only $23.5 \%$ of the studies showed the level of schooling of the patients, the health condition of the victims in $64.7 \%$ of the cases presented with fractures and traumas. The most traumatized region was the face in $41.1 \%$ of the cases, in relation to the type of injury in $17 \%$ of cases it was due to trauma and $6.4 \%$ was due to bruises. In $24.5 \%$ of the studies the victims made use of alcoholic beverages and $29.4 \%$ did not use the helmet at the time of the accident. Conclusion: In view of this it is necessary that studies that identify or trace the profile of these individuals, determining type of accidents and commitment, be performed in a more detailed way, to produce necessary subsidies for the planning of public health policies and promotion of preventive measures.

Descriptors: Accidents, Traffic; Epidemiology; Facial Injuries.

\section{Resumo}

Objetivo: O objetivo do presente estudo foi descrever o perfil de pacientes com trauma facial por acidentes motociclísticos no Brasil. Métodos: O estudo consistiu em uma revisão integrativa da literatura, sendo consultadas as bases de dados Scientific Electronic Library Online (SciELO) e National Library of Medicine (PubMed), utilizando os seguintes descritores: Acidentes de Trânsito Epidemiologia, e Traumatismos Faciais de acordo com as terminologias consultadas nos Descritores em Ciências da Saúde (DECS-BIREME ) A pesquisa considerou as publicações de 1995 a 2015. Resultados: Quanto ao sexo masculino, os jovens do sexo masculino predominaram em 100\% dos estudos. Apenas 23,5\% dos estudos mostraram o nível de escolaridade dos pacientes, o estado de saúde das vítimas em 64,7\% dos casos, apresentavam fraturas e traumas. A região mais traumatizada foi a face em $41,1 \%$ dos casos, em relação ao tipo de lesão em $17 \%$ dos casos foi por trauma e $6,4 \%$ por hematomas. Em $24,5 \%$ dos estudos, as vítimas fizeram uso de bebidas alcoólicas e $29,4 \%$ não usavam capacete no momento do acidente. Conclusão: Diante disso, é necessário que estudos que identifiquem ou traçam o perfil desses indivíduos, determinando tipos de acidentes e comprometimentos, sejam realizados de forma mais detalhada, para produzir subsídios necessários ao planejamento de políticas públicas de saúde e promoção de medidas preventivas.

Descritores: Acidentes de Trânsito; Epidemiologia; Traumatismos Faciais.

\section{INTRODUCCIÓN}

El accidente de tránsito se define como todo ocurrido casual y no intencional que ocurre en vía pública, causante de víctimas con lesiones físicas y/o emocionales, por choque y/o colisiones ${ }^{1}$. Según un informe de la Organización Mundial de la Salud (OMS), los accidentes de tránsito afectan a cerca de 1,25 millones de personas al año, siendo la mitad de las víctimas de personas en grupos más vulnerables como: motociclista, ciclistas y peatones.

En Brasil el desarrollo industrial proporcionó un crecimiento bastante rápido en la flota automovilística, con destaque la flota de motos, esa flota tuvo un registro de 20.593.246 motos reguladas hasta junio de 2016 según el Departamento Nacional 
de Tráfico (DENATRAN). Estos números pueden ser justificados por la facilidad de adquisición y el menor costo de mantenimiento de este tipo de vehículo, sin embargo, hay una gran desventaja en lo que se refiere a la seguridad y protección de los usuarios ${ }^{2-4}$.

El alto índice de mortalidad en accidentes con motocicletas en Brasil es considerado un problema de salud pública, siendo catalogado por la Clasificación Internacional de Enfermedades (CID) como causa externa ${ }^{5}$. Con las principales causas la conducción bajo el efecto de alcohol y alta velocidad, mientras que el número de personas afectadas por lesiones y traumas considerando la totalidad de accidentes automovilísticos en general es de aproximadamente 50 millones al año $\mathrm{a}^{2,6}$. Un trauma en la cara puede acometer además de tejido blando y hueso, el cerebro, ojos, dentición y senos de la cara, pudiendo caracterizar una agresión letal ${ }^{7}$. Los traumas que alcanzan la región maxilofacial están entre los más habituales en los centros de tratamiento de urgencia y emergencia ${ }^{8}$.

En este sentido, la investigación, buscó por medio de una revisión integrativa trazar el perfil de los pacientes con traumas de cara derivados de accidentes con motocicleta en Brasil. Con el fin de fomentar el conocimiento científico acerca del tema y contribuir con la planificación de políticas públicas de salud en la prevención de agravios y promoción en salud.

\section{MATERIAL Y METODO}

El presente estudio se caracteriza como una revisión integrativa, observacional, descriptiva, cuantitativa, transversal, con datos secundarios, que tuvo como propósito trazar el perfil de los pacientes con trauma de cara derivado de accidentes con motocicletas en Brasil.

La población estudiada fue compuesta por artículos publicados, a cerca de los accidentes con motocicletas en Brasil. La muestra final fue compuesta por los artículos relacionados al trauma de cara derivados de accidentes con motocicletas, publicados entre los años 1995 a 2015, pudiendo ser del tipo relato de caso, serie de casos, y / o investigaciones epidemiológicas y estuvieron disponibles en su totalidad para la lectura. Los artículos sin estas características fueron excluidos.

Los datos fueron recolectados a través de la observación de artículos publicados en Brasil en el período de 20 años, disponibles en las bases de datos de consulta Scientific Electronic Library on-line (SciELO) y National Library of Medicine (PubMed). La elección final de los trabajos fue dirigida, en la que se eligieron tres palabras claves, previamente verificadas en los Descriptores en Ciencias de la Salud (DESC-BIREME) para ordenar la elección de los artículos, los cuales se hagan saber: Accidentes de Tráfico, Epidemiología, Trauma de cara. Después de la selección de los artículos, los mismos investigadores leyeron cada uno de los artículos y realizaron el análisis de los datos contenidos en éstos. El proceso de selección de la muestra final se encuentra dispuesto en el diagrama de flujo (Figura 1).

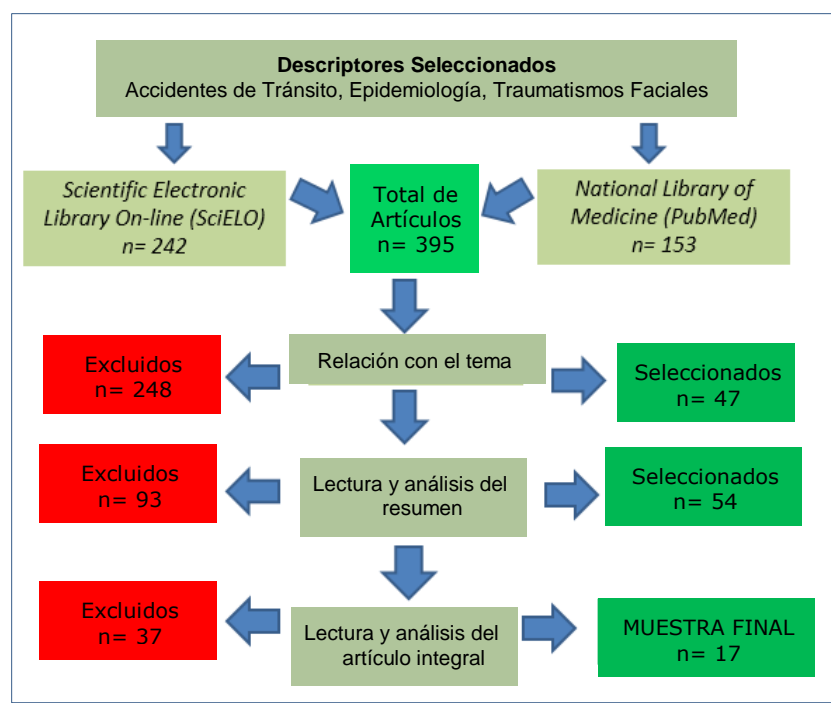

Figura. 1 - Diagrama de flujo de selección de artículos.

Se ha buscado datos como: tema/objetivo del artículo, año de la publicación, región de origen de la investigación, estado de origen de la investigación, numero de la muestra, sexo predominante, raza/etnia, edad/grupo de edad predominante, grado de instrucción/escolaridad, el tiempo/día del accidente, el tipo de accidente predominante, las condiciones de salud de la víctima (lesión/trauma/muerte), región traumatizada/lesionada, tipo de lesión facial, $\mathrm{CNH}$ : posee o puerta, uso del alcohol, uso del casco . Todos los datos fueron dispuestos y organizados en una ficha específica, creada para tal investigación (Cuadro 1).

\begin{tabular}{|l||}
\hline \hline FICHA ESPECíFICA PARA FICHAMENTO DE LOS ARTÍCULOS \\
\hline \hline Título del artículo: \\
\hline \hline Autores: \\
\hline \hline Año de publicación: \\
\hline \hline Región de origen de la investigación: \\
\hline \hline Estado de origen de la investigación: \\
\hline \hline Muestra (n): \\
\hline \hline Sexo predominante: \\
\hline \hline Raza/Origen étnico: \\
\hline \hline Edad/Franja etaria predominante: \\
\hline \hline Grado de instrucción/escolaridad: \\
\hline \hline Período/Tiempo/Día del accidente: \\
\hline \hline Tipo de accidente predominante: \\
\hline \hline Condiciones de salud de la víctima (lesión/trauma o muerte): \\
\hline \hline Región traumatizada/lesionada: \\
\hline \hline Tipo de lesión facial: \\
\hline \hline CHN: ¿Posee o puerta? \\
\hline \hline ¿Uso de alcohol? \\
\hline \hline ¿Uso de casco? \\
\hline
\end{tabular}

Cuadro 1: Perfil de las víctimas de accidentes con motocicletas en Brasil.

Todos los artículos de la muestra final fueron leídos y los datos fueron analizados de la siguiente forma: tema principal y objetivo (categorizados en dos grupos, los relacionados y los no relacionados). 
Para el año de publicación se incluyeron sólo el período entre 1995 a 2015; en relación a la región donde se realizaron las encuestas fueron: noreste, sureste, norte, sur, centro-oeste; para los estados de origen el criterio fue los 26 estados más el Distrito Federal; ya la muestra numérica de los artículos fueron incluidas como muestreo para nuestra investigación; el sexo clasificado como femenino o masculino; las etnias fueron incluidas como feoderma, leucoderma y melanoderma; la edad considerada entre 10 y 60 años; ya el grado de instrucción fueron incluidos enseñanza básica incompleta / completa, enseñanza media completa / incompleta y superior completo / incompleto; período / tiempo / día del trauma fueron dados en número de días horas o período del accidente; tipos de accidentes automotrices con implicación motociclística o solamente motociclista; las condiciones del paciente fueron de acuerdo con lesión, trauma y muerte; las regiones traumatizadas fueron consideradas aquellas que tienen relación sólo con la cara; los tipos de lesiones se incluyeron en los artículos que definen el tipo de lesión; que posee o no posee $\mathrm{CNH}$; uso o no uso del alcohol durante el accidente; también se verificaron los que declararon sí o no para el uso del casco. Los artículos que no se ajustaron a los criterios de inclusión se excluyeron.

Los resultados obtenidos se organizaron en una base de datos informatizada con la ayuda del paquete estadístico Statistical Package for the Social Sciences (SPSS) (v.20). Se realizó análisis de la distribución de los datos de forma descriptiva exponiendo los resultados en porcentajes, frecuencias simples, los cuales fueron discutidos en la revisión integrativa.

El presente trabajo fue desarrollado con datos secundarios y datos que no pueden en modo alguno perjudicar, producir maleficios o incomodidad a los individuos, por lo tanto, respeta los principios éticos de la Resolución 466/2012 del Consejo Nacional de Salud (CNS) que regula las investigaciones involucrando seres humanos, ya que el objeto principal del estudio es la literatura, aunque ésta retrate datos provenientes inicialmente de individuos encuestados.

\section{RESULTADOS}

El alto índice de mortalidad por accidentes motociclísticos en Brasil es considerado un problema de salud pública, estando catalogado por la Clasificación Internacional de Enfermedades (CID), como en Brasil se registraron en el año 2014 cerca de 156.942 óbitos por causa externas, donde la CID considera accidentes motocíclicos como causas externas, según el Ministerio de Salud (MS) y el Sistema de Información de Mortalidad (SIM). El desarrollo industrial subsidió un crecimiento considerable en la flota automovilística en Brasil, destacándose la flota de motos, que atizaron un registro de 20.593.246 unidades con placa en Brasil, según DENATRAN- Departamento Nacional de Tráfico en junio de 2016. Estas cifras son justificadas por la facilidad de adquisición y menor costo de mantenimiento, sin embargo, hay una gran desventaja en lo que se refiere a la seguridad y protección de los usuarios $^{2-4,6,9}$.

Según un informe de la Organización Mundial de la Salud (OMS), los accidentes de tránsito, que sufrieron víctimas de tránsito, sufrieron la muerte de 1,25 millones de personas al año, siendo la mitad de las víctimas más vulnerables como: Motociclista $23 \%$, peatón $22 \%$ y ciclista $4 \%$. En el caso de que se produzca un accidente en el que se produzca un accidente de tránsito en el que se produzca un accidente, h esta probabilidad aumenta al $60 \%$ y la utilización del uso del casco que puede reducir en un $40 \%$ el riesgo de muerte y el $70 \%$ de las lesiones graves. El número de personas afectadas por lesiones y traumas por la totalidad de accidentes es de aproximadamente 50 millones al año ${ }^{2,6}$.

Según la Organización Mundial de la Salud $(\mathrm{OMS})^{10}$, las leyes sobre seguridad mejoran el comportamiento de los usuarios y reducen los números de colisiones, de lesiones y de muertes en el tráfico, especialmente las leyes relacionadas con los cinco principales factores de riesgo, que son un exceso de velocidad, conducción bajo el efecto de alcohol, no uso de cascos por los motociclistas, no uso de cinturones de seguridad y sistemas de retención de niños.

Las concesionarias y las industrias de motocicletas equipararon los valores gastados con transporte público mensuales con las parcelas de consorcios y financiamientos, facilitando la adquisición de estos vehículos por la clase C y D, dando una alternativa mejor a esas personas, pues transporte colectivo presenta incomodidad, ineficacia y ineficacia inseguridad ${ }^{10}$. El aumento del número de motocicletas, en los últimos años, sigue el número de accidentes, especialmente en carreteras municipales, donde el número de accidentes fatales por ese tipo de transporte superó a todos los demás vehículos. Entre 1998 y 2008 el número de accidentes motociclísticos aumentó en el $754 \%{ }^{11}$.

Los accidentes involucrados en motocicletas en Brasil causan grandes consecuencias en la morbilidad y mortalidad, en la población que afecta principalmente a adultos jóvenes, que son económicamente productivos, lo que acarrea directamente mayores gastos en la salud y pérdidas en la economía del país. En lo que se refiere a la mortalidad de los datos facilitados por los sistemas oficiales de información sanitaria en 2006, muestran en el grupo de 20 a 39 años una tasa de mortalidad por accidentes de motociclismo es de aproximadamente 7,3 por cada 100 mil habitantes, 
una tasa de defunción el mismo motivo es del 19,8\%. En cuanto a la morbilidad, el $76,7 \%$ de las internaciones fueron por accidente de tránsito, el $28,2 \%$ causadas por accidentes con motocicletas ${ }^{6}$.

La gran incidencia de jóvenes varones, puede ser justificada por la falta de experiencia en la conducción, además de la impulsividad, la alta confianza, la audacia, el arrojo, el atrevimiento, la prueba de límites, el coraje, lo que son características más prevalentes en los hombres jóvenes ${ }^{11}$.

En cuanto a la mortalidad, en Brasil no hay datos específicos, tal vez por falta de recolección de datos en relación a traumas, esa recolección sería de fundamental importancia para orientar acciones de abordaje y tratamiento de víctimas con ese tipo de trauma, donde un sistema informatizado sería el " ideal para el análisis de los datos, donde el resultado de esas informaciones sería fundamental para obtener las incidencias ${ }^{12}$.

En cuanto al perfil de las víctimas de accidentes motociclistas, el sexo masculino fue más prevalente en los estudios, con la mayor prevalencia en individuos jóvenes con edad entre 19 y 40 años. Sólo algunos de los estudios presentaron el nivel de escolaridad de los pacientes, y cuando verificado las víctimas presentaban apenas el $1{ }^{\circ}$ grado de instrucción (Tabla 1) $)^{2-9,12-22}$.

Tabla 1. Perfil de los accidentados según sexo, etnicidad, edad e escolaridad

\begin{tabular}{|c|c|c|c|c|}
\hline Autor/Año & Sexo & Etnicidad & Edad & Escolaridad \\
\hline $\begin{array}{c}\text { Brasileiro et al. }^{2} \\
2010\end{array}$ & Masculino & $\begin{array}{l}\text { La mayoría } \\
\text { con feodermia }\end{array}$ & $21-30$ & Fundamental \\
\hline $\begin{array}{c}\text { Gomes et al. } 4 \\
2014 \\
\end{array}$ & Masculino & $\mathrm{x}$ & $15-25$ & Fundamental \\
\hline $\begin{array}{c}\text { Legay et al. }{ }^{6} \\
2007\end{array}$ & Masculino & $\mathrm{x}$ & $20-24$ & Fundamental \\
\hline Silva et al.7 2011 & Masculino & $\mathrm{X}$ & $30-35$ & $\mathrm{X}$ \\
\hline $\begin{array}{c}\text { Vasconcelos et al. }{ }^{8} \\
2014\end{array}$ & Masculino & $\mathrm{x}$ & 20-35 & $\mathrm{X}$ \\
\hline $\begin{array}{c}\text { Shoeller et al. }{ }^{9} \\
2012\end{array}$ & Masculino & $\mathrm{x}$ & $25-34$ & $\mathrm{x}$ \\
\hline $\begin{array}{c}\text { Grawryzewski et al. }{ }^{13} \\
2009\end{array}$ & Masculino & $\mathrm{x}$ & $20-29$ & $\mathrm{x}$ \\
\hline $\begin{array}{c}\text { Macêdo et al. }{ }^{14} \\
2007\end{array}$ & Masculino & $\mathrm{x}$ & $21-40$ & $\mathrm{X}$ \\
\hline $\begin{array}{c}\text { Macêdo et al. }{ }^{15} \\
2012\end{array}$ & Masculino & $\mathrm{x}$ & $18-25$ & $\mathrm{X}$ \\
\hline $\begin{array}{c}\text { Marin-León et al. }{ }^{16} \\
2012\end{array}$ & Masculino & $\mathrm{x}$ & $15-39$ & $\mathrm{x}$ \\
\hline $\begin{array}{c}\text { Montovani et al. }{ }^{17} \\
2006\end{array}$ & Masculino & $\mathrm{x}$ & $\mathrm{X}$ & $\mathrm{X}$ \\
\hline $\begin{array}{c}\text { Rezende Neta et al. }{ }^{18} \\
2012 \text { sera } 18\end{array}$ & Masculino & $\mathrm{x}$ & $11-40$ & $\mathrm{X}$ \\
\hline $\begin{array}{c}\text { Sarmento et al. }{ }^{19} \\
2007\end{array}$ & Masculino & $\mathrm{x}$ & $21-30$ & $\mathrm{X}$ \\
\hline $\begin{array}{c}\text { Silva et al. }{ }^{20} \\
2015 \\
\end{array}$ & Masculino & $\mathrm{x}$ & $19-29$ & $\mathrm{X}$ \\
\hline $\begin{array}{c}\text { Trevisol et al. }^{21} \\
2012 \\
\end{array}$ & Masculino & $\mathrm{x}$ & $14,9-35,3$ & $\mathrm{X}$ \\
\hline $\begin{array}{c}\text { Vieira }^{22} \\
2011 \\
\end{array}$ & Masculino & $\mathrm{x}$ & 27,78 & $\mathrm{x}$ \\
\hline $\begin{array}{l}\text { Wulkan et al. }{ }^{23} \\
2005\end{array}$ & Masculino & $\mathrm{x}$ & 20-39 & $\mathrm{x}$ \\
\hline
\end{tabular}

En los artículos seccionados la región más traumatizada fue la cara en el $41,1 \%$ de los casos y en relación al tipo de lesión $17 \%$ de los casos ocurridos trauma, el 6,4\% contusiones y el $76,4 \%$ de los trabajos no presentaron resultados para el tipo de lesión facial (Tabla 2).
Tabla 2. Perfil de los accidentados condiciones da salud da víctima, región del trauma e tipo de la lesión facial

\begin{tabular}{|c|c|c|c|}
\hline Autor/Año & $\begin{array}{l}\text { Condiciones da } \\
\text { salud de la victima }\end{array}$ & $\begin{array}{l}\text { Región del } \\
\text { trauma }\end{array}$ & $\begin{array}{c}\text { Tipo de la } \\
\text { lesión facial }\end{array}$ \\
\hline $\begin{array}{c}\text { Brasileiro et al. }^{2} \\
2010\end{array}$ & Traumas y fraturas & Dientes y cara & $\begin{array}{c}\text { Trauma } \\
\text { dentoalveolar }\end{array}$ \\
\hline $\begin{array}{c}\text { Gomes et al. } 4 \\
2014\end{array}$ & $\mathrm{x}$ & $\mathrm{x}$ & $\mathrm{x}$ \\
\hline $\begin{array}{c}\text { Legay et al. } \\
2007\end{array}$ & $\begin{array}{l}\text { Corte, laceración y } \\
\text { fractura }\end{array}$ & $\mathrm{X}$ & $\mathrm{X}$ \\
\hline Silva et al. 72011 & Trauma & Cara & $\begin{array}{c}\text { Fractura de la } \\
\text { mandíbula }\end{array}$ \\
\hline $\begin{array}{c}\text { Vasconcelos et al. } \\
2014 \\
\end{array}$ & $\mathrm{x}$ & $\begin{array}{c}\text { Tercio inferior } \\
\text { de la cara }\end{array}$ & $\mathrm{x}$ \\
\hline $\begin{array}{c}\text { Shoeller et al. }{ }^{9} \\
2012 \\
\end{array}$ & Trauma en la cabeza & Cráneo & $\mathrm{x}$ \\
\hline $\begin{array}{c}\text { Grawryzewski et al. }{ }^{13} \\
2009\end{array}$ & $\begin{array}{l}\text { Fractura, trauma en la } \\
\text { cabeza }\end{array}$ & Cara & $\mathrm{X}$ \\
\hline $\begin{array}{c}\text { Macêdo et al. }{ }^{14} \\
2007\end{array}$ & $\mathrm{x}$ & Cara & $\begin{array}{l}\text { Lesión y } \\
\text { fractura }\end{array}$ \\
\hline $\begin{array}{c}\text { Macêdo et al. }{ }^{15} \\
2012\end{array}$ & $\mathrm{x}$ & $\mathrm{x}$ & $\mathrm{x}$ \\
\hline $\begin{array}{c}\text { Marin-León et al. }{ }^{16} \\
2012 \\
\end{array}$ & $\mathrm{x}$ & $\mathrm{x}$ & $\mathrm{x}$ \\
\hline $\begin{array}{c}\text { Montovani et al. }{ }^{17} \\
2006\end{array}$ & Trauma & $\begin{array}{c}\text { Mandíbula y } \\
\text { cigoma }\end{array}$ & $\mathrm{X}$ \\
\hline $\begin{array}{c}\text { Rezende Neta et al. }{ }^{18} \\
2012\end{array}$ & Trauma & Cara & $\mathrm{x}$ \\
\hline $\begin{array}{c}\text { Sarmento et al. }{ }^{19} \\
2007\end{array}$ & Trauma & Cara & $\mathrm{X}$ \\
\hline $\begin{array}{c}\text { Silva et al. }{ }^{20} \\
2015\end{array}$ & Fractura & Cara & $\mathrm{x}$ \\
\hline $\begin{array}{c}\text { Trevisol et al. }{ }^{21} \\
2012\end{array}$ & Fractura & Cara & $\mathrm{x}$ \\
\hline $\begin{array}{c}\text { Vieira }^{22} \\
2011 \\
\end{array}$ & $\begin{array}{l}\text { Excoriaciones en la } \\
\text { cabeza }\end{array}$ & $\begin{array}{c}\text { Cabeza, cara y } \\
\text { cuello }\end{array}$ & $\mathrm{x}$ \\
\hline $\begin{array}{l}\text { Wulkan et al. }{ }^{23} \\
2005\end{array}$ & $\mathrm{x}$ & $\mathrm{x}$ & Contusiones \\
\hline
\end{tabular}

En el estudio de Brasileiro et al. (2010), el $67 \%$ de las víctimas no portaban $\mathrm{CNH}$,

Los datos obtenidos cuanto el uso de documento del conductor (permiso para conducir), el uso de bebidas alcohólicas y el uso de casco se muestran en la Tabla 3.

Tabla 3. Caracterización de las condiciones de las víctimas en el momento de los accidentes con motocicletas en Brasil

\begin{tabular}{|c|c|c|c|}
\hline Autor/Año & $\begin{array}{c}\text { No llevaba } \\
\text { permiso }\end{array}$ & $\begin{array}{l}\text { Bajo el efecto } \\
\text { del alcohol }\end{array}$ & $\begin{array}{l}\text { No utilizaba } \\
\text { casco }\end{array}$ \\
\hline $\begin{array}{c}\text { Brasileiro et al. }^{2} \\
2010\end{array}$ & $\begin{array}{l}\text { No llevaba } \\
\text { permiso }\end{array}$ & Mayoría & Mayoría \\
\hline $\begin{array}{c}\text { Gomes et al. } 4 \\
2014\end{array}$ & $\mathrm{X}$ & $\mathrm{x}$ & Mayoría \\
\hline $\begin{array}{c}\text { Legay et al. } \\
2007\end{array}$ & $\mathrm{x}$ & Mayoría & $\mathrm{x}$ \\
\hline Silva et al. ${ }^{7} 2011$ & $\mathrm{x}$ & Mayoría & Mayoría \\
\hline $\begin{array}{c}\text { Vasconcelos et al. }^{8} \\
2014\end{array}$ & $\mathrm{X}$ & $\mathrm{x}$ & $\mathrm{x}$ \\
\hline $\begin{array}{c}\text { Shoeller et al. }{ }^{9} \\
2012\end{array}$ & $\mathrm{x}$ & $\mathrm{x}$ & $\mathrm{x}$ \\
\hline $\begin{array}{c}\text { Grawryzewski et al. }{ }^{13} \\
2009 \text { sera } 13\end{array}$ & $\mathrm{x}$ & $\mathrm{x}$ & $\mathrm{x}$ \\
\hline $\begin{array}{c}\text { Macêdo et al. }{ }^{14} \\
2007\end{array}$ & $\mathrm{x}$ & $\mathrm{x}$ & $\mathrm{x}$ \\
\hline $\begin{array}{l}\text { Macêdo et al. } .^{15} \\
2012\end{array}$ & $\mathrm{x}$ & $\mathrm{x}$ & $\mathrm{x}$ \\
\hline $\begin{array}{c}\text { Marin-León et al. }{ }^{16} \\
2012\end{array}$ & $\mathrm{X}$ & $\mathrm{x}$ & $\mathrm{x}$ \\
\hline $\begin{array}{c}\text { Montovani et al. }{ }^{17} \\
2006\end{array}$ & $\mathrm{X}$ & $\mathrm{X}$ & $\mathrm{x}$ \\
\hline $\begin{array}{l}\text { Rezende Neta et al. }{ }^{18} \\
2012\end{array}$ & $\mathrm{x}$ & $\mathrm{x}$ & Mayoría \\
\hline $\begin{array}{c}\text { Sarmento et al. }{ }^{19} \\
2007\end{array}$ & $\mathrm{x}$ & $\mathrm{x}$ & $\mathrm{x}$ \\
\hline $\begin{array}{c}\text { Silva et al. }{ }^{20} \\
201\end{array}$ & $\mathrm{x}$ & $\mathrm{x}$ & $\mathrm{x}$ \\
\hline $\begin{array}{c}\text { Trevisol et al. }{ }^{21} \\
2012\end{array}$ & $\mathrm{x}$ & $\mathrm{x}$ & $\mathrm{x}$ \\
\hline $\begin{array}{l}\text { Vieira }^{22} \\
2011\end{array}$ & $\mathrm{x}$ & Mayoría & Mayoría \\
\hline $\begin{array}{l}\text { Wulkan et al. }{ }^{23} \\
2005\end{array}$ & $\mathrm{x}$ & $\mathrm{x}$ & $\mathrm{x}$ \\
\hline
\end{tabular}




\section{DISCUSIÓN}

El trauma facial es reconocido como una enfermedad pandémica, según datos de la OMS, una agresión en la cara puede acometer más allá de tejido blando y hueso, el cerebro, ojos, dentición y senos de la cara, pudiendo causar una agresión letal ${ }^{7}$. Adicionalmente, los traumas que alcanzan la región buco-maxilo-facial están entre los más habituales en los centros de tratamiento de urgencia y emergencia ${ }^{8}$.

Las fracturas maxilofaciales contribuyen alarmantemente a la morbimortalidad, desfiguración permanente, consecuencias emocionales y funcionales, invalidez y costos en la salud. Estudios muestran que los accidentes de tránsito fueron responsables por cerca del $45 \%$ de las causas de fracturas maxilofaciales. El 9\% de estos traumas se debió a accidentes de motociclismo ${ }^{3,7}$. En un estudio similar, Brasileiro et al. ${ }^{2}$ constataron que el $22 \%$ de los casos de accidentes con motocicletas, los pacientes presentaron fracturas mandibulares ${ }^{2}$.

En lo que se refiere a la gravedad de la lesión traumática, asociados a las regiones de la cabeza más acometidas son: fractura de mandíbula, fractura nasal, laceración de la córnea, fractura de diente, laceración del nervio óptico y fractura del tipo Lefort $\mathrm{II}^{7}$. El trauma en la región de cabeza y cuello figura como una de las principales causas de muertes en Brasil, y el accidente de tránsito contribuye a que esta ocurrencia se convierta en un serio problema, pues además de acarrear en muertes, causan muchas secuelas y mayores costos directos e indirectos ${ }^{9}$. La principal causa de muerte en pacientes con trauma bucomaxilofaciales son lesiones en el Sistema Nervioso Central (SNC), seguido por choque hemorrágico y traumas en regiones específicas ${ }^{11}$.

En el estudio de Brasileiro et al. ${ }^{2}$, la mayor prevalencia de los accidentes con motocicletas fue para pacientes del género masculino con 127 hombres de un total de 145 casos; alcoholizados en el $71,7 \%$ de los casos; sin el uso del casco, el 77,9\%; las personas con una edad media de 28,6 años; donde el principal motivo fue el desequilibrio del 78,6\%; con impacto de la cara directamente al suelo $71,7 \%$; sin $\mathrm{CNH}$ en el $67 \%$ de los casos.

En el estudio presentado por Vasconcelos et al. $^{8}$ de los 248 pacientes analizados, $197(79,4 \%)$ eran hombres y $51(20,6 \%)$ mujeres. El grupo de edad más afectado fue de 20 a 35 años de edad (45,7\%). El accidente de tránsito que rodea la moto representó el $61,5 \%$ de los casos. Los sitios anatómicos de la cara en que fue acometido el trauma facial se dividieron en tercios: el tercio inferior $(57,7 \%)$ y medio $(46,4 \%)$.

En el estudio de Macêdo et al. ${ }^{15}$ realizado el año de 2007 fueron evaluados 771 pacientes, de este $72,8 \%$ eran del sexo masculino, el $52 \%$ de los pacientes presentaban edades comprendidas entre 21 y 40 años. En cuanto a la etiología la principal fue agresión, seguidos de accidentes de vehículos y motos en el $14,9 \%$ de los casos, caída de bicicleta en el $13,2 \%$. Entre las lesiones de cara $75,1 \%$ de los casos acometieron tejidos blandos, y el $24,9 \%$ de los casos ocurrieron fracturas, de estas fracturas las mayores causas fueron la agresión física y accidentes de tránsito. En relación a los locales de las fracturas faciales prevaleció la nariz en el 76,8\% de los casos, seguida del zigoma con el 9,6\% de los casos.

El sexo masculino y el grupo de edad de individuos jóvenes prevalecieron en el $100 \%$ de los estudios ${ }^{2,4,6,7-9,13-23}$. Sólo el $23,5 \%$ de los estudios presentaron el nivel de escolaridad de los pacientes ${ }^{2,46}$, donde se presentaron bajo nivel de escolaridad. En el estudio de Brasileiro et al. ${ }^{2}$ y Gomes et al. ${ }^{4}$ la mayoría de las víctimas presentaron enseñanza fundamental completa. En el estudio de Legay et al. ${ }^{6}$, las víctimas presentaron de 9-11 años de estudios.

En un estudio Sarmento et al. ${ }^{19}$ realizaron una investigación con datos de accidentes entre 2002 y 2006, en el Hospital Regional de Campina Grande donde 13.822 pacientes fueron atendidos entre estos 691 ingresaron en el servicio de maxilofacial. Estos pacientes presentaron en el $8,5 \%$ fracturas mandibulares, eran del sexo masculino en el $83,1 \%$ de los casos y con edades más prevalentes entre 21 y 30 años. El período de la noche registró mayores ocurrencias el 30,5\% de los casos. El principal factor etiológico para fracturas de mandíbula fue, caída de la propia altura $28,8 \%$, seguido por accidentes de moto y violencias interpersonales, ambos con el $23,7 \%$ de los casos. Los días que ocurrieron más atendimientos fueron: sábado, Domingo y lunes ambos con el 20,3\%.

En cuanto la condición de salud de las víctimas, en los estudios seleccionados se observó que en el $64,7 \%$ de los casos los pacientes se presentaran con fracturas y traumasLa condición de salud de las víctimas en el 64,7\% de los casos se presentó con fracturas y traumas faciales $2,6,7,9,13,17-21,23$ y el $35,2 \%$ de los estudios no presentó resultados para la condición de salud de las víctimas ${ }^{4,8,14-16,23}$. La región más traumatizada fue la cara en el $41,1 \%$ de los casos ${ }^{2,7,8,13,15,18-22}$ y en relación al tipo de lesión $17 \%$ de los casos se dio por trauma facial ${ }^{2,7,15}$, el $6,4 \%$ se dio por contusiones ${ }^{23}$.

Los estudios seleccionados no presentaron resultados cuanto el uso de documento del conductor (permiso para conducir). En el 23,5\% del estudios relataran que las víctimas estaban bajo el efecto del alcohol $^{2,6,7,22}$. Luego, $76,5 \%$ de los estudios no presentaron resultados para el uso de bebidas alcohólicas $^{4,8,9,13-21,23}$. En el 29,4\% de los estudios seleccionados la mayoría de las víctimas no utilizaban el casco en el momento del accidente $e^{2,4,7,18,22}$ y en el $70,6 \%$ de los estudios no fueran fornecidos datos cuanto el uso de casco en momento del accidente ${ }^{8.9 \cdot 13-21,23}$. 
Para solucionar los problemas de tránsito involucrando a los motociclistas, a través de la prevención se incluye uso de los equipos de protección individual, mantenimiento preventivo del vehículo, comportamiento del usuario y mejora en las vías públicas ${ }^{11}$. En el caso específico de los motociclistas la prevención como el uso de cascos, chaquetas, guantes, educación, medidas legales y protección pasiva sería el factor clave para disminuir los riesgos de accidente ${ }^{22}$.

\section{CONCLUSIÓN}

Con base nos resultados obtenidos puede concluirse que el sexo masculino y el grupo de edad de los individuos jóvenes prevaleció en todos los estudios. Sólo algunos estudios presentaron el nivel de escolaridad de los pacientes, donde éstos presentaron bajo nivel de escolaridad. La condición de salud de las víctimas en su mayoría se presentó con fracturas y traumas faciales. La región más traumatizada fue la cara y en relación al tipo de lesión la mayoría se dio por trauma facial. La mayoría de las víctimas estaban bajo el efecto de bebidas alcohólicas y no utilizaban el casco en el momento del accidente. Por lo tanto, es necesario que estudios que identifiquen o tracen el perfil de estos individuos determinando tipo de accidentes y comprometimiento, sean realizados de forma más detallada, principalmente en relación al uso de documento del conductor (permiso para conducir), etnia y grado de instrucción, donde la mayoría de los estudios no presentaron resultados, para producir subsidios necesarios para la planificación de políticas públicas en salud y promoción de medidas preventivas.

\section{REFERENCIAS}

1. Santos LNM. Acidentes de trânsito: uma análise a partir de publicações bibliográficas. $61^{\circ}$ Congresso Brasileiro de Enfermagem. FortalezaCE, 2009. Disponível em: <www.abeneventos. com.br/ anais_61cben/ files/ 00462.pdf>.Acesso em: 10 out. 2016.

2. Brasileiro BF, Vieira JM, Silveira CES. Avaliação de traumatismos faciais por acidentes motociclísticos em Aracaju SE. Rev Cir Traumatol Buco-Maxilo-fac Camaragibe. 2010;10(2):97-104.

3. Furtado DR. Perfil do trauma de face em pacientes vítimas de acidentes motociclísticos [dissertação]. Londrina: Universidade Estadual de Londrina - UEL; 2015.

4. Gomes LS, Santos YA, Dourado SBPB, Coêlho DMM, Moura MEB. Perfil das vítimas de acidentes motociclísticos admitidas nas terapias intensivas de um hospital público. Rev Enferm UFPE. 2014;8(7):2004-12.

5. Costa MJC, Mangueira JA. Perfil epidemiológico de ocorrências no trânsito no Brasil - revisão integrativa. Sanare Sobral. 2014;13(2):110-16.

6. Legay LF, Santos AS, Lovisi GM, Aguiar JS, Borges JC, Mesquita RM et al. Acidentes de transporte envolvendo motocicletas perfil epidemiológico das vítimas de três capitais de estados brasileiros. Epidemiol Serv Saúde. 2012;21(2):283-92.

7. Silva JJL, Lima AAAS, Melo IFS, Maia RCL, Pinheiro Filho TRC. Trauma facial análise de 194 casos. Rev Bras Cir Plást. 2011;26(1):37-41.

8. Vasconcelos BG, Silva LAC, Silva Júnior AF, Mohn Neto CR, Pereira CM. Perfil epidemiológico dos pacientes com fraturas faciais atendidos em um hospital de Goiânia-Goiás. J Health Sci Inst. 2014;32(3):241-45.

9. Schoeller SD, Bonetti A, Silva GA, Rocha A, Gelbcke FL, Khan P. Características das vítimas de acidentes motociclisticos atendidas em um centro de reabilitação de referência estadual do sul do Brasil. Acta Fisiátr. 2011;18(3):141-45.

10. Who. World Health Organization. Report Global On The Status Safety Road 2015. Goals of road safety and objectives sustainable development. Genebra. OMS. 2015.

11.Seerig LM. Motociclistas: Perfil, prevalência de uso da moto e acidentes de trânsito- Estudo de base populacional [dissertação]. Pelotas: Universidade Católica de Pelotas - UCPEL; 2012.

12.Trajano AD, Pereira BM, Fraga GP. Epidemiology of in-hospital trauma deaths in a Brazilian university hospital. BMC Emerg Med. 2014;14:22.

13.Gawryszewski VP, Coelho HMM, Scarpelini S, Zan R, Jorge MHPM, Rodrigues EMS. Perfil dos atendimentos a acidentes de transporte terrestre por serviços de emergência em São Paulo. Rev Saúde Pública. 2009; 43(2):275-82.

14.Macêdo JLS, Camargo LM, Almeida PF, Rosa SC. Mudança etiológica do trauma de face de pacientes atendidos no pronto socorro de cirurgia plástica do Distrito Federal. Rev Bras Cir Plást. 2007;22(4): 209-12.

15. Macêdo DWM, Oliveira FPA. Epidemiologia de acidentes urbanos com atendimento móvel de urgência, Santarém, PA, maio a setembro de 2009. Rev Saúde e Desenvolvimento. 2012;22(4):209-12.

16. Marin-León L, Belon AP, Barros MBA, Almeida SDM, Restitutti MC. Tendência dos acidentes de trânsito em Campinas, São Paulo, Brasil: importância crescente dos motociclistas. Cad. Saúde Pública. 2012;28(1):39-51.

17. Montovani JC, Campos LMP, Gomes MA, Moares VRS, Ferreira FD, Nogueira FD. Etiologia e incidência das fraturas faciais em 
adultos e crianças: experiência em 513 casos. Rev Bras Otorrinolaringol. 2006; 72(2):235-41.

18. Rezende Neta DS, Alves AKS, Leão GM, Araújo AA. Perfil das ocorrências de politrauma em condutores motociclísticos atendidos pelo SAMU de Teresina-PI. Rev bras enferm. 2012; 65(6):936-41.

19. Sarmento DJS, Cavalcanti AL, Santos JA. Características e distribuição das fraturas mandibulares por causas externas: estudo retrospectivo. Pesq Bras Odontoped Clin Integr. 2007;7(2):139-44.

20.Silva MGP, Silva VL, Lima MLLT. Lesões craniofaciais decorrentes de acidentes por motocicleta: uma revisão integrativa. Rev CEFAC. 2015;17(5):1689-97.

21. Trevisol DJ, Bohm R L, Vinholes DB. Perfil epidemiológico dos pacientes vítimas de acidentes de trânsito atendidos no serviço de emergência do Hospital Nossa Senhora da Conceição em Tubarão, Santa Catarina. Scientia Medica. 2012;22(3):148-52.

22. Vieira RCA, Hora EC, Oliveira DV, Vaez AC. Levantamento epidemiológico dos acidentes motociclísticos atendidos em um Centro de Referência ao Trauma de Sergipe. Rev Esc Enferm USP. 2011;45(6):1359-63.

23. Wulkan NM, Parreira Jr JG, Botter DA. Epidemiologia do trauma facial Rev Assoc Med Bras. 2005;51(5):290-95.

\section{CONFLICTO DE INTERESES}

Los autores declaran no tener conflictos de intereses.

AUTOR PARA CONTACTAR

Jefferson David Melo de Matos

matosjefferson19@gmail.com

Enviado em 17/09/2018

Aceptado em 12/03/2019 